\title{
Inferring the 3D Standing Spine Posture from 2D Radiographs
}

\author{
Amirhossein Bayat ${ }^{1,2} \star$, Anjany Sekuboyina ${ }^{1,2 \star}$, Johannes C. Paetzold ${ }^{1}$, \\ Christian Payer ${ }^{3}$, Darko Stern ${ }^{3}$, Martin Urschler ${ }^{4}$, Jan S. Kirschke ${ }^{2 \star \star}$, and \\ Bjoern H. Menze $\mathrm{e}^{1 \star \star}$ \\ 1 Department of Informatics, Technical University of Munich, Germany \\ 2 Department of Neuroradiology, Klinikum rechts der Isar, Germany \\ 3 Institute of Computer Graphics and Vision, Graz University of Technology, Austria \\ 4 School of Computer Science, University of Auckland, New Zealand \\ amir.bayat@tum.de
}

\begin{abstract}
The treatment of degenerative spinal disorders requires an understanding of the individual spinal anatomy and curvature in 3D. An upright spinal pose (i.e. standing) under natural weight bearing is crucial for such bio-mechanical analysis. 3D volumetric imaging modalities (e.g. CT and MRI) are performed in patients lying down. On the other hand, radiographs are captured in an upright pose, but result in $2 \mathrm{D}$ projections. This work aims to integrate the two realms, i.e. it combines the upright spinal curvature from radiographs with the $3 \mathrm{D}$ vertebral shape from CT imaging for synthesizing an upright 3D model of spine, loaded naturally. Specifically, we propose a novel neural network architecture working vertebra-wise, termed Trans Vert, which takes orthogonal 2D radiographs and infers the spine's 3D posture. We validate our architecture on digitally reconstructed radiographs, achieving a 3D reconstruction Dice of $95.52 \%$, indicating an almost perfect $2 \mathrm{D}$-to3D domain translation. Deploying our model on clinical radiographs, we successfully synthesise full-3D, upright, patient-specific spine models for the first time.
\end{abstract}

Keywords: 3D reconstruction - Fully Convolutional Neworks · Spine posture $\cdot$ Digitally Reconstructed Radiographs

\section{Introduction}

A biomechanical study of spine and its load analysis in upright standing position is an active research topic, especially in cases of spine disorders [1. Most common approaches for load estimation on the spine either use a general computational model of the spine for all patients or acquire subject-specific models from magnetic resonance imaging (MRI) or computed tomography (CT) [3]. While these typical 3D image acquisition schemes capture rich 3D anatomical

\footnotetext{
* equal contribution

$\star \star$ Joint supervising authors
} 


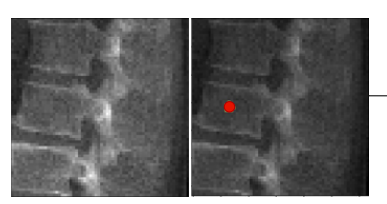
Sagittal DRR vertebra \& Centroid
annotation

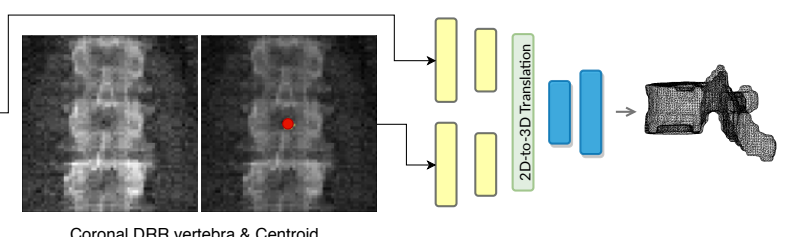

Coronal DRR vertebra \& Centroid

Fig. 1: Overview of 2D image to 3D shape translation. The network inputs are $2 \mathrm{D}$ orthogonal view vertebrae patches and the centroid indicating the vertebra of interest.

information, they require the patient to be in a prone or supine position (lying on one's chest or back), for imaging the spine. But, analysis of the spine's shape and vertebral arrangement needs to be done in a physiologically upright standing position under weight bearing, making $2 \mathrm{D}$ plain radiographs a de facto choice. A combination of both these worlds is of clinical interest to fully assess the biomechanical situation, i.e. to capture patient-specific complex pathological spinal arrangement in a standing position and with $3 \mathrm{D}$ information $[2] 3]$.

In literature, numerous registration-based methods have been proposed for relating $2 \mathrm{D}$ radiographs with $3 \mathrm{D} \mathrm{CT}$ or MR images. In [8], the authors propose a rough manual registration of $3 \mathrm{D}$ data to $2 \mathrm{D}$ sagittal radiographs for the lumbar vertebrae. For the same purpose, in 4, manual annotations of the vertebral bodies are used as guideline for measuring the vertebral orientations in upright standing position. These methods are time and manual-labour-intensive and thus prone to error. Moreover, both these works use only the sagittal radiographs for vertebra positioning, while ignoring the coronal reformation which is a strong indicator of the spine's natural curvature. Aiming at this objective, 9] introduced an automatic 3D-2D spine registration algorithm, where the authors propose a multi-stage optimization-based registration method by introducing a metric for comparing a CT projection with a radiograph. However, this metric is hand-crafted, parameter-heavy, and is not learning-based, thus limiting its generalizability. In [10, the 3D shape of the spine is reconstructed using a biplanar X-ray device called 'EOS'. Hindering its applicability is the high device cost and the lack of its presence in a clinical routine. Recently the problem of reconstructing 3D shapes given 2D images have been explored using deep learning approaches. An approach closest to ours was proposed by Ying et al. [12, where they introduce a deep neural network to synthesize 3D CT images given orthogonal radiographs using adversarial networks. However, this model is highly memory intensive and fails to synthesise smaller anatomies like vertebrae in 3D. Moreover, it has been evaluated only on digitally reconstructed radiographs (DRR), and its clinical applicability remains to be validated. 
Motivation The problem of 3D reconstruction of a spine in an anatomically upright position from $2 \mathrm{D}$ radiograph images relies on retrieving information from radiographs, which are 2D projections of a 3D object. Spine's sagittal reformation captures crucial information in the form of the vertebral body's and process' shape and its orientation around the sagittal (left-right) axis. However, its orientation around the cranio-caudal and anterior-posterior axes is obfuscated (cf. Fig. 1). This information is available when combining sagittal with coronal reformations (or lateral with a.p. radiographs). Motivated by this, we propose a fully-supervised, computationally efficient, and registration-free approach combining sagittal and coronal 2D images to synthesise the vertebra's 3D shape model. Specifically:

- We introduce a novel fully convolutional network (FCN) architecture for fusing orthogonal radiographs to generate $3 \mathrm{D}$ shapes.

- We identify an approach for training the network on synthetically generated radiographs from CT, being supervised by the CT's 3D vertebral masks.

- Validating our approach, we achieve dice score of $\mathbf{9 5 . 5 2 \%}$ on digitally reconstructed radiographs. We also successfully reconstruct 3D, patient-specific spine models on real clinical radiographs.

\section{Methods}

Generating 3D shapes from 2D information is an ill-posed problem. For solving this, we utilize information from two orthogonal radiographs and an annotation on the vertebra of interest while relying on the shape prior learnt by the network.

\subsection{TransVert: Translating 2D information to 3D shapes}

The network performing 2D-to-3D synthesis needs to address the following requirements: First, it needs to appropriately combine information in the sagittal and coronal projections to recover 3D information. Second, recovering 3D shapes from 2D projections is inherently an ill-posed problem, requiring incorporation of prior knowledge. Lastly, the size of certain vertebra (towards the scan's periphery) is larger in radiographs compared to their true size due to the cone-beam of gamma-ray source. This effect should be negated when reconstructing the 3D model, i.e. the mapping should not be purely image-based. We address these requirements by proposing the Trans Vert architecture.

Overview TransVert takes four 2D inputs, the sagittal and coronal vertebral image patches and their corresponding annotation images indicating the vertebraof-interest (VOI). Denoting the 2D vertebral sagittal and coronal reformations by $x_{s}$ and $x_{c}$, and their corresponding VOI annotation by $y_{s}$ and $y_{c}$, we desire the vertebra's full-body $3 \mathrm{D}$ shape, $\mathbf{y}$, as a discrete voxel-map:

$$
\mathbf{y}=G\left(x_{s}, x_{c}, y_{s}, y_{c}\right)
$$




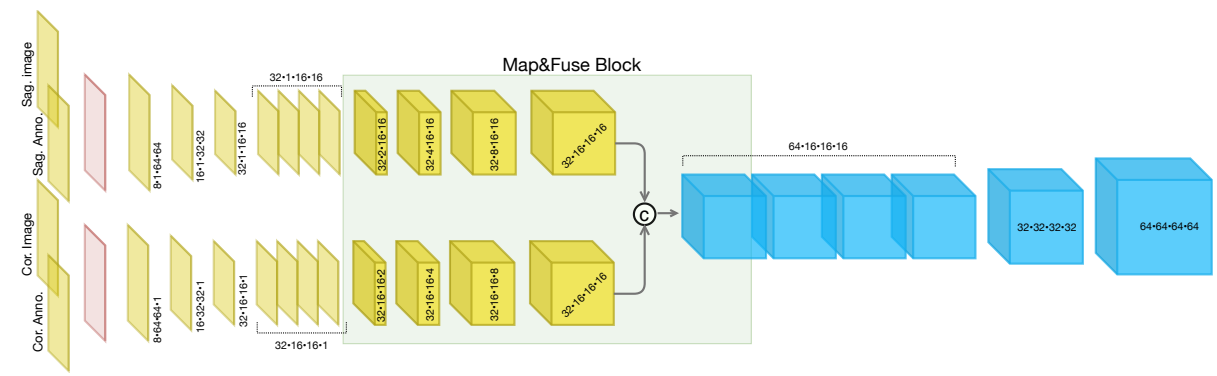

Fig. 2: Architecture of TransVert. Our model is composed of sagittal and coronal 2D encoders (self-attention module in red), a 'map\&fuse' block, and a 3D decoder.

where $G$ denotes the mapping performed by TransVert. In our case, the VOI-annotation image is obtained by placing a disc of radius 1 around the vertebral centroid. In Section 3, we analyze denser annotation choices (vertebral body and full vertebral masks). Ideally, training the TransVert mapping requires radiograph images and their corresponding 'real world' 3D spine models. However, this correspondence does not exist and is, in fact, the problem we intend to solve. Thus, TransVert is trained on sagittal and coronal digitally reconstructed radiographs (DRR) constructed from CT images. It is supervised by the corresponding CT images' voxel-level, vertebral segmentation masks. As DRRs are similar in appearance to real radiographs, a DRR-trained TransVert architecture paired with a robust training regime, can be readily deployed on clinical radiographs.

Architecture TransVert consists of three blocks: a 2D sagittal encoder, a 2D coronal encoder, and a 3D decoder. The three blocks are combined by a 'map\&fuse' block. Refer to Fig. 2 for a detailed illustration. The map\&fuse block is responsible for mapping $2 \mathrm{D}$ representations of each the sagittal and coronal views into intermediate 3D latent representations followed by fusing them into a single 3D representation by channel-wise concatenation. This representation is then decoded into a viable $3 \mathrm{D}$ voxelized representation by the decoder. Note that the intermediate 3D representation is constructed from orthogonal views. Therefore, map\&fuse block consists of anisotropic convolutions, with an anisotropy along the dimensions that need to be expanded. For example: the anterior-posterior dimension needs to be expanded for a coronal view. Consequently, the convolutional strides and padding directions are orthogonal for each of the view. At the network encoders' input, the vertebral images and VOI-annotations are combined using a self-attention layer. It was empirically observed that the attention mechanism yielded a better performance than a naive fusion by concatenating them as multiple channels. 
Loss Using solely a regression loss leads to converging to a local optimum where a mean (or median) shape is predicted, especially in the highly varying regions of the vertebra such as the vertebral processes. This is rectified by augmenting the loss with an adversarial component which checks the validity of a prediction at a global level. Therefore, TransVert is trained in a fully supervised manner by optimizing a combination of an $\ell_{1}$ distance-based regression loss and an adversarial loss based on the least-squared GAN (LSGAN, [5]). Formally, the TransVert and the Discriminator combination is trained by minimizing the following losses:

$$
\begin{gathered}
\mathcal{L}_{G}=\alpha_{G}\left\|\mathbf{y}-G\left(x_{s}, x_{c}, y_{s}, y_{c}\right)\right\|_{1}+\alpha_{D}\left(D\left(G\left(x_{s}, x_{c}, y_{s}, y_{c}\right)\right)-1\right)^{2} \text { and } \\
\mathcal{L}_{D}=(D(\mathbf{y})-1)^{2}+D\left(G\left(x_{s}, x_{c}, y_{s}, y_{c}\right)\right)^{2}
\end{gathered}
$$

where $D$ represents the discriminator network and $G$ represents the TranVert. $\alpha_{G}$ and $\alpha_{D}$ are weights of loss terms and fixed to $\alpha_{G}=10$ and $\alpha_{D}=0.1$. Note that $\mathbf{y}$ is binary valued containing $\{0, i\}$, where $i \in\{8,9, \ldots 24\}$ denotes the vertebral index from T1 to L5. Forcing the network to predict the vertebral index implicitly incorporates an additional prior relating the shape to the vertebral index. Details about the discriminator architecture and the adversarial training regime are provided in the supplemental material. The network is implemented with Pytorch framework on a Quadro P6000 GPU. It is trained till convergence using an Adam optimizer with initial learning rate is 0.0001 .

\section{Results}

In this section, we describe the creation of DRRs, present an ablative study quantitatively analyzing the contribution of various architectural components, compare various VOI-annotation types, and finally deploy TransVert on real clinical radiographs.

\subsection{Data}

Recall that TransVert works with two data modalities: it is trained on DRRs extracted from CT images while being supervised by their corresponding 3D segmentation mask, and it is deployed on clinical radiographs.

CT data We work with two datasets: a publicly available dataset for lung nodule detection with 800 chest CT scans [13], and an in-house dataset with 154 CT scans. In all, we work with $\sim 12 k$ vertebrae split $5: 1$ forming the training and validation set, reporting 3 -fold cross validated results. Note that very few lumbar vertebrae are visible in [13] as it is lung-centred.

Data Preparation: The CT scans are segmented using [11] and the generated masks are validated by an experienced neuro-radiologist in order to consider 
only accurate ones for the study. These vertebral masks are used for supervision. Generation of the corresponding digitally reconstructed radiographs (DRR) is performed using a ray-casting approach [7, wherein a line is drawn from the radiation source (focal point) to every single pixel on the digitally reconstructed radiographs (DRR) image and the integral of the $\mathrm{CT}$ intensities over this line are calculated. Parameters for this generation include the radiation source-todetector $(=180 \mathrm{~cm}$ in this work $)$ and the source-to-object distance $(=150 \mathrm{~cm}$ here). Post the generation of the sagittal and coronal digitally reconstructed radiographs (DRR), patches of size $64 \times 64$ are extracted around each vertebral centroid, constituting the image input to TransVert. The second input, viz. the VOI-annotation, can be extracted from the projected segmentation mask.

Clinical radiographs We clinically validate TransVert on real long standing radiographs in corresponding lateral and anterior-posterior (a.p.) projections obtained in 30 patients. Acquisition parameters such as source-to-detector and source-to-object distances were similar to those used for DRR generation. Vertebral centroids needed for the VOI-annotations were automatically generated on both views using [] .

Data normalization TransVert is trained on DRRs and tested on clinical radiographs. These data modalities have different intensity ranges, requiring normalization. We observe that z-score normalization works well, i.e. $\mathcal{I}=\left(\mathcal{I}-\mu_{\mathcal{I}}\right) / \sigma_{\mathcal{I}}$, where $\mu_{\mathcal{I}}$ and $\sigma_{\mathcal{I}}$ are the mean and standard deviation of the image $\mathcal{I}$.

\section{$3.2 \quad$ Experiments}

We perform three sets of experiments validating our proposed approach, aimed at analysing the architectural aspects of TransVert, the data fed into it, and finally its applicability in a clinical setting. Note that a quantitative comparison with the ground truth can be performed only in experiments dealing with DRRs and CT images. Performance evaluation of various settings is compared by computing Dice coefficient and Hausdorff Distance between the predicted 3D vertebral mask and its ground truth from the CT mask.

Analysing TransVert's architecture The proposed architecture for TransVert consists of the following architectural choices: fusion of sagittal and coronal views, anisotropic convolutions in the map\&fuse block, a self-attention layer combining the image and the VOI-annotation, and finally, an adversarial component on the loss function. An ablative study over these components is reported in Table 1. First, do we need two views?. For this, we evaluate the performance of a model that tries to reconstruct 3D shape from only a sagittal image. Next, do we need anisotropic convolutions?. For this, we compare two versions of map\&fuse: one with a simple outer product for combining the orthogonal views (Naive View-Fusion) and one with the proposed anisotropic convolutions (TransVert). 


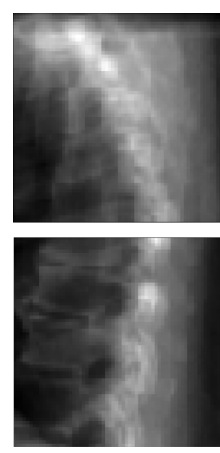

Input image
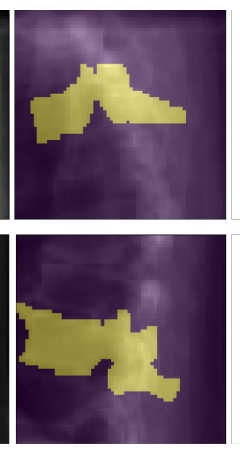

Ground truth overlay
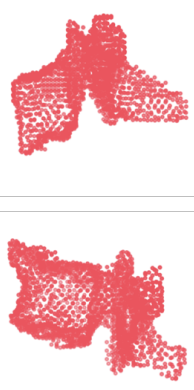

Ground truth model
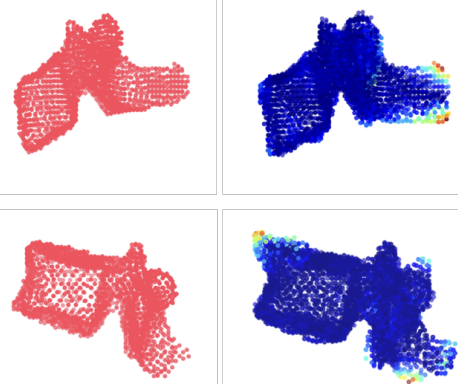

Predicted model

Chamfer Distance Map

Fig. 3: Shape modelling with TransVert on DRRs: First column indicates the image input. Second and third columns visualise the ground truth (GT) vertebral mask and the fourth visualises the predicted 3D shape model. Last column shows an overlayed Chamfer distance map between point clouds of GT and prediction.

\begin{tabular}{c|c|c}
\hline Setup & Dice (\%) & Hausdorff $(\mathrm{mm})$ \\
\hline Sagittal only & 88.40 & 7.43 \\
Naive View-Fusion (Outer Product) & 92.59 & 6.45 \\
TransVert & 94.75 & 5.75 \\
TransVert + Self Attn. & 95.31 & 5.27 \\
TransVert + SelfAttn + Adv. & 95.52 & 5.11 \\
\hline
\end{tabular}

Table 1: Architectural ablative study: The performance progressively improves with addition of each component. (Vertebral centroids are the VOI-annotations here.

Observe that a simple fusion of views already outperforms a 'sagittal only' reconstruction. Also, anisotropic convolutions outperform fusion of views using outer-products. This can be attributed to the 2D-to-3D learning component involved in the latter. Lastly, do we need the bells 83 whistles on top of Trans Vert? Observe that incorporating the self-attention layer in the encoders and an adversarial training regime progressively improved performance, resulting in a Dice of $95.5 \%$ and a Hausdorff Distance of $5.11 \mathrm{~mm}$. Fig. 3 illustrates the 3D shape models reconstructed using the proposed architecture. Extracting a point cloud (with 2048 points) from these shapes, we also illustrate a point-wise Chamfer distance map. Observe that a vertebra's posterior region (vertebral process) is hardly visible in the image inputs. Despite this, TransVert is capable of recovering the process, albeit with a certain disagreement between the prediction and ground truth. 
Analysing VOI-annotation type Recall that alongside the image input, TransVert requires an auxiliary input indicating the vertebra of interest. We argue that a vertebral centroid suffices. In this study we show that our choice of vertebral centroid performs at a level comparable to a far denser full-vertebra annotation as reported in Table 2. We compare our centoids-to-vertebra $(\mathrm{C} 2 \mathrm{~V})$ setup to two other, denser annotations: one where the vertebral body is annotated in the DRR (B2V) and one where the full vertebral body is annotated $(\mathrm{V} 2 \mathrm{~V})$. As baseline, we include a setup without any VOI-annotation as an auxiliary input. Note that including the annotation input offers approximately $20 \%$ improvement in the mean Dice coefficient. Observe that a most dense V2V annotations and our $\mathrm{C} 2 \mathrm{~V}$ annotations perform comparably with only $<1 \%$ difference. Therefore, $\mathrm{C} 2 \mathrm{~V}$ is an obvious choice owing to the ease of marking centroids, more so because of existing automated labelling approaches.

Table 2: VOI annotation study: Performance drop from a denser $(\mathrm{V} 2 \mathrm{~V})$ to a sparser annotation $(\mathrm{C} 2 \mathrm{~V})$ is minor, while annotation effort decreases man-

\begin{tabular}{c|c|c}
\hline Input & Dice (\%) & Hausdorff $(\mathrm{mm})$ \\
\hline No annotation & 76.44 & 14.74 \\
V2V & 96.24 & 4.18 \\
B2V & 95.67 & 4.95 \\
C2V & 95.31 & 5.27 \\
\hline
\end{tabular}
ifold.

2D-to-3D translation in clinical radiographs TransVert works with individual vertebral images and their centroids. A 3D model of the spine can be constructed by stacking the predicted 3D vertebrae models at their corresponding 3D centroid locations. Vertebra's position along the axial and coronal axes is obtained from the sagittal reformation and its sagittal position from the coronal reformation. Fig. 4 illustrates the results of this process. The top row visualises a 3D spine reconstruction based on 2D DRRs and compares it with the ground truth. More importantly, the bottom row depicts a successful deployment of TransVert in reconstructing the $3 \mathrm{D}$, patient-specific posture of upright standing spine. Note that no 3D ground truth spine model exists for these cases. We visualise the $2 \mathrm{D}$ overlay of the segmentation on the radiographs, and the sagittal and coronal view of its 3D shape model, the former overlaid on the radiograph too. Observe that the 3D model's posture matches with that of the radiographs.

\section{Conclusion}

We propose TransVert, a novel architecture trained to infer a full-3D spine model from 2D sagittal and coronal radiographs and sparse centroid annotations. We identify an approach to train TransVert on DRRs in a fully-supervised manner. Along with an ablative study on TransVert's architectural components, we show a successful use case of deploying it on a real-world clinical radiograph. 

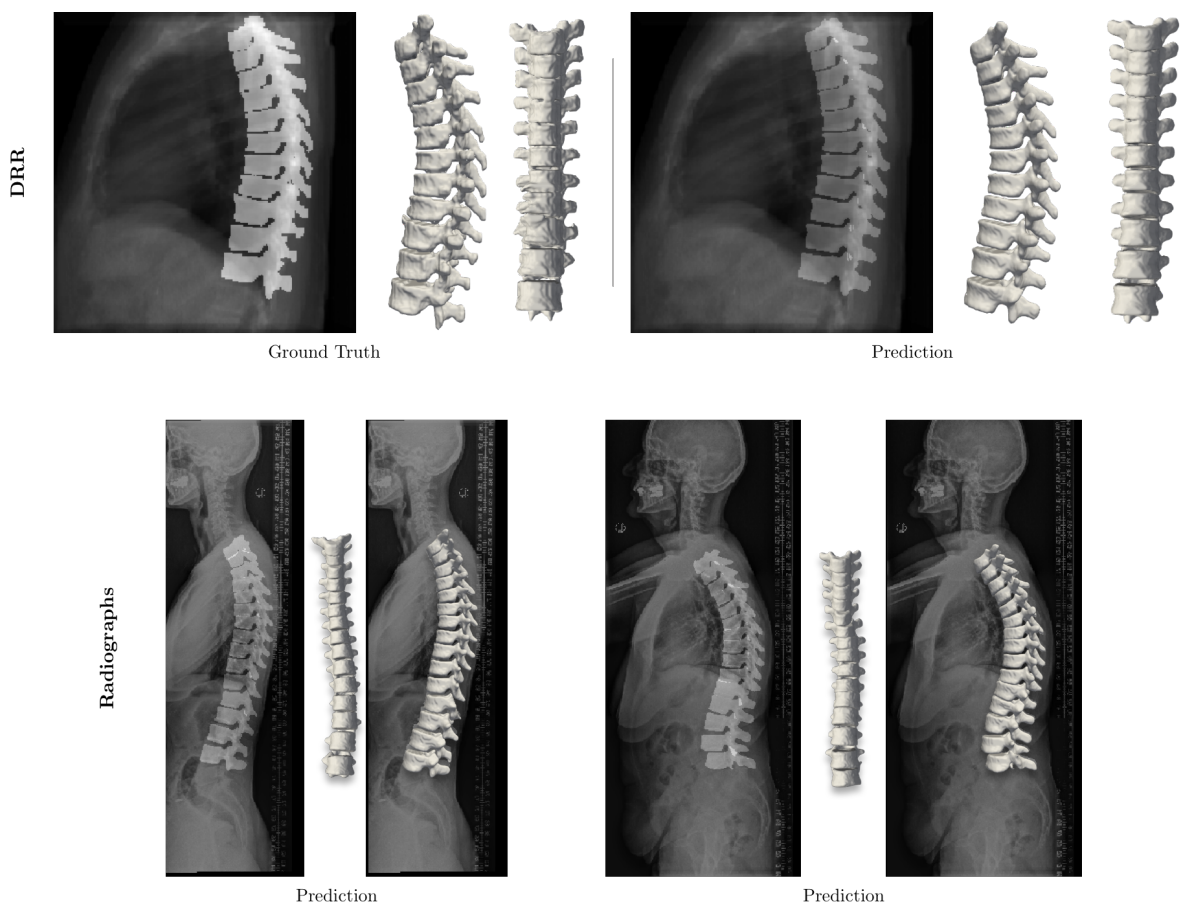

Fig. 4: Full 3D spine models: (Top row) Comparison of a DRR-based spine model reconstruction with its CT ground truth mask. (Bottom row) 3D patient-specific spine models constructed from real clinical radiographs. (Best viewed by zooming in.)

Acknowledgements This work was funded from the European Research Council (ERC) under the European Union's Horizon 2020 research and innovation programme (GA637164-iBack-ERC-2014-STG).

\section{References}

1. Dreischarf, M, et al. Estimation of loads on human lumbar spine: a review of in vivo and computational model studies. Journal of biomechanics 49.6 (2016): 833-845.

2. El Ouaaid, Z, et al. Effect of changes in orientation and position of external loads on trunk muscle activity and kinematics in upright standing. Journal of Electromyography and Kinesiology 24.3 (2014): 387-393.

3. Akhavanfar, M. H., et al. Obesity and spinal loads; a combined MR imaging and subject-specific modeling investigation. Journal of biomechanics 70 (2018): 102-112.

4. Bauer S, et al. Effects of individual spine curvatures-a comparative study with the help of computer modelling. Biomedical Engineering/Biomedizinische Technik. 2012 Sep 1;57(SI-1 Track-O):132-5. 
5. Mao X, el al.: Least squares generative adversarial networks. In: IEEE ICCV. pp. 2794-2802. (2017)

6. Bayat, A., et al. Vertebral Labelling in Radiographs: Learning a Coordinate Corrector to Enforce Spinal Shape. Computational Methods and Clinical Applications for Spine Imaging: 39.

7. Staub, D., et al. A digitally reconstructed radiograph algorithm calculated from first principles. Medical physics 40.1 (2013): 011902.

8. Eskandari, A. H., et al. Subject-specific 2D/3D image registration and kinematicsdriven musculoskeletal model of the spine. Journal of biomechanics 57 (2017): 18-26.

9. Ketcha, M. D., et al. Multi-stage 3D-2D registration for correction of anatomical deformation in image-guided spine surgery. Physics in Medicine \& Biology 62.11 (2017): 4604

10. Humbert, L., et al. 3D reconstruction of the spine from biplanar X-rays using parametric models based on transversal and longitudinal inferences. Medical engineering \& physics 31.6 (2009): 681-687.

11. Sekuboyina, Anjany, et al. VerSe: A Vertebrae Labelling and Segmentation Benchmark. arXiv preprint arXiv:2001.09193 (2020).

12. Ying, X., et al. X2CT-GAN: reconstructing CT from biplanar X-rays with generative adversarial networks. IEEE CVPR. 2019.

13. Armato III, et al. The lung image database consortium (LIDC) and image database resource initiative (IDRI): a completed reference database of lung nodules on CT scans. Medical physics 38.2 (2011): 915-931. 